\title{
Information Dissemination Control Algorithm of Ecological Changes in the New Media Communication Environment
}

\author{
Juanjuan Huang $\mathbb{D}^{1,2}$ \\ ${ }^{1}$ Nanjing Forestry University, Nanjing 210037, China \\ ${ }^{2}$ Nanjing University Jinling College, Nanjing 210042, China \\ Correspondence should be addressed to Juanjuan Huang; 030259@jlxy.nju.edu.cn
}

Received 1 July 2021; Accepted 31 July 2021; Published 10 August 2021

Academic Editor: Fazlullah Khan

Copyright (c) 2021 Juanjuan Huang. This is an open access article distributed under the Creative Commons Attribution License, which permits unrestricted use, distribution, and reproduction in any medium, provided the original work is properly cited.

Information dissemination has been a crucial social process, especially in modern information-centric societies; it has become one of the most critical ones. Social networks create a route for information dissemination with the use of social influence mechanisms. In order to improve the effectiveness of information dissemination control and influence the maximization of ecological change in the modern media communication environment, this paper combines the current network information dissemination methods and analyzes the community network model and the network dissemination states for spreading information. We propose an improved algorithm for information dissemination control of ecological changes in the new media dissemination environment. The fuzzy control algorithm is employed as the core algorithm to improve and optimize the information dissemination network model. Furthermore, the mobile-assisted patch distribution mechanism is used to repair all the infected nodes in the network, and the information interaction form is analyzed to develop the corresponding system model structure and build the information traceability process. The simulation results verify the feasibility of using the proposed algorithm to control effectively the information dissemination in the ecological changes of the new media environment. The proposed algorithm can meet the ecological information control needs of new media dissemination.

\section{Introduction}

Information dissemination has been a crucial social process, particularly in recent information-centric societies. It has become one of the most critical ones. Most of the commercial communication infrastructures have been originally established in the past 20 years mostly to allow the transfer of various types of information. Diverse types of information are disseminating in human societies, especially through the computer and communication networks. These different types of information include news, educational, healthcare, financial, and military information. With the development of today's social science and technology, the development of multimedia, and the maturity of intelligent networks, these have gradually developed into new trends in the Internet. The Internet has had a great impact on human lives. All the information we need can be found on the Internet, and people's lives have gradually become inseparable from the network. Internet resources have expanded the wealth of space and time that people perceive and disseminate information and improved their active learning initiative [1]. People can get news, educational, healthcare, financial, and other information from the network at any time and from any place. Spreading valuable information on the network can meet the requirements of people not only of education but also of all sectors and ages to get information related to healthcare, financial, military, and climate and other aspects of learning. Many large-scale online social network sites, such as Facebook, Twitter, and Friendster, have become popular because they effectively connect people and bring together small and disconnected offline social networks. Moreover, they also become a huge dissemination and marketing platform, allowing information and ideas to influence a large population in a short period. 
New media channels have built the fastest and most extensive social networking platform for the audience to disseminate news and other information. However, unlike the rigorous requirements for the dissemination of news and scientific and technological knowledge, new social networking platforms and channels may pursue sensational effects or emphasize relevance to life and dramatic plots, which directly affect the content of scientific and technological communication [2]. The study of the content of science and technology communication ecology in the new media era is based on the perspectives of relevance and integrity and dynamics and balance in ecological thinking to infiltrate ecology from the study of the interaction between organisms and the environment into other fields such as humanities and social sciences. Science and technology communication is a kind of communication practice activity, which focuses on the relationship between the main communication media and the communication content. It refers to the process in which scientific and technological information is used as the main content of communication to spread between the subject and the audience in the social environment and pursue a dynamic balance through the medium. In this sense, the communication of science and technology is ecological [1]. The study associates the media ecology tradition with the concept of the ecosystem, which was initially used in business economics. In an ecosystem economy, organizations work in close collaboration and share information more freely. One of the most basic themes of the media ecology tradition is how different public and private societies work and how to disseminate electronic and digital media. Therefore, by comparing the similarities between the science and technology communication ecosystem and the natural ecosystem, analyzing the scope and complexity of the science and technology communication scenarios from the perspective of ecology, and revealing the nature and laws of science and technology communication, the real problems of the ecological imbalance of science and technology communication in the new media era can be better investigated [3]. Encouraged by this analysis, this study proposed an improved algorithm for the information dissemination control of ecological changes in the new media dissemination environment. Moreover, the fuzzy control algorithm was employed as the kernel algorithm to improve the system, build the corresponding frame structure, and analyze the system performance with experimental research.

The rest of the paper is organized as follows. Section 2 presents the related works. Section 3 provides a discussion about different methods for membership function selection in fuzzy set theory. Section 4 illustrates the proposed information dissemination control algorithm. In Section 5, the performance results are presented, and Section 6 is about the conclusion.

\section{Related Works}

The impact of social networks on people's lives has increased significantly. The social influence for information dissemination acts as an inspiring force, governing the dissemination of the information in the network. When studying influence maximization, the communication model is the most basic problem, and the problem of maximizing social influence is explored through the information dissemination model. Therefore, the selection of communication models is also an important research issue in the subject of influence maximization. The most classic ones of the information dissemination models that study influence maximization are the independent cascade (IC) model [4] and the linear threshold (LT) model [5]. Based on the independent cascade model and the linear threshold model, the general cascade model, the general threshold model, and the decreasing cascade propagation model have been successively proposed [6]. More and Lingam [7] proposed an improved independent cascade model and performed a comparison of the different approaches to compute activation probabilities of nodes in a social network. The path method (PM) was applied to compute the exact value of the activation probabilities but has an approximated method called SSS-Noself, which was computed by changing the existing SteadyStateSpread algorithm, based on fixed-point computation, to achieve better accuracy. Kemp et al. [8] proposed a greedy method for searching " $K$ " influential network nodes out of all existing nodes. They provided an approximation-guaranteed solution for the greedy algorithm. They designed an analysis structure for finding the seed nodes. This framework was depended upon submodular functions. A triggering model was also proposed to show that their proposed approximation algorithm worked better as compared with other known node selection strategies in social networks. However, the drawback of this algorithm is its efficiency. Moreover, an important part of their greedy algorithm is to compute the spread of influence based on a seed set, which turns out to be a challenging task. Kimura and Saito [9] developed influence cascade models based on the shortest path and designed an efficient algorithm to compute influence spread under these models. However, the models do not directly address the efficiency issue of the greedy algorithms for the cascaded models. The authors of [10] formulated the problem of information dissemination and influence maximization as the discrete optimization problem. A social network is represented as a graph with vertices showing individuals and edges representing connections between two individuals. Influence is broadcasted in the network based on the stochastic cascade model. Three cascade models, including the independent cascade model, the linear threshold model, and the weight cascade model, were considered for influence maximization.

A fast maximum influence arborescence (FMIA) model was proposed in [11]. The model uses the local tree structure of each node in the graph to adjust efficiently the top-k influencers in an evolving network. The method recursively predicts only the affected seed nodes due to active updates in the influence graph and then replaces them with more suitable ones. The method is effective in a variety of information dissemination models and influence maximization techniques. Pazura et al. [12] presented an improved model of the LT model, called competitive linear threshold (CLT model). In this model, each edge has two weights, plus, 
and minus. Based on the CLT model, the problem of maximum influence blocking is proposed to study how an object blocks the information transmission of competitors.

To improve the efficiency of the greedy algorithm, a new algorithm CELF++ was proposed based on the cost-effective lazy forward (CELF) algorithm. It augments the naive greedy algorithm to maximize the influence in social networks. The CELF++ algorithm makes use of the property of submodularity of the spread function to avoid excessive computations of marginal gains incurred by CELF. It was reported that on real-world social network data sets, the CELF++ works effectively and efficiently, resulting in significant improvements in terms of both running time and the average number of node lookups [13].

A new susceptible-infected-recovered (SIR) model was proposed to model the social network diffusion [5] based on various theories such as bond percolation [14]. Likewise, graph evolution parameters, such as shrinking diameters, and densification were examined for modeling information dissemination and influence maximization in social networks [15]. Based on global social network metrics, such as closeness centrality and betweenness centrality, a semilocal centrality metric was presented to design an effective ranking method. This semilocal centrality metric along with the SIR epidemic model was used to assess the application of the diffusion model by considering the parameters such as the rate of influence spread and the number of infected nodes [16]. Social networks were investigated for quantifying user influence, and they dealt with web semantics to learn about influence in heterogeneous social networks [17]. Social influence was further employed for the study of human dynamics and human behavior [18]. In this study, a new algorithm is proposed for information dissemination and influence maximization of ecological changes in the new media communication environment. The fuzzy control algorithm is employed as the core algorithm to improve and optimize the network node model.

\section{Information Dissemination Control Algorithm Based on Fuzzy Theory}

Fuzzy set theory is an extension of the classical concept of a set. The basis of this theory is the fuzzy set, which is a set that does not have clearly defined limits and can contain elements only to some degree in which elements can have a certain degree of membership [19]. Therefore, suitable functions are used called membership functions that determine the membership degree of each element in a fuzzy set. The membership functions perform a vigorous role in the overall performance of fuzzy representation. The membership functions are the building blocks of fuzzy set theory. The only condition a membership function must satisfy is that it must vary between 0 and 1 [20]. The degree of membership of an element $x$ relative to set $A$ can be represented in the form of a function. Set $A$ is called a fuzzy set, and $\mu_{A}$ is called the membership function of the fuzzy set. For a fuzzy set $A$, it can be characterized by its membership function. The closer the value $\mu_{A}$ is to 0 ; the lower is the degree that the element $x$ belongs to set $A$. On the contrary, the closer the value of $\mu_{A}$ is to 1 ; the higher is the degree that the element $x$ belongs to set
$A$. In extreme cases, the value $\mu_{A}$ is only 0 or 1 . At this time, $\mu_{A}$ is an ordinary traditional function, and set $\mathrm{A}$ is an ordinary set [19]. There are many ways to represent the fuzzy sets. In general, it can be expressed as follows:

$$
A=\left\{\frac{\mu_{A}(X)}{X}, x \in X\right\}
$$

For a fuzzy set containing multiple elements, $A$ can also be represented using the following equation:

$$
A=\sum_{i=1}^{n} \frac{\mu_{A}\left(X_{i}\right)}{X_{i}}, \quad x \in X
$$

The commonly used methods for determining the membership function are given in the following sections.

3.1. Expert Determination Method. This method is used to determine the degree of membership value through subjective knowledge or personal experience, especially the expert's experience. This method is mainly used in the case of discrete elements in the universe of discourse. The membership degree values usually obtained have low credibility, but they are also closer to the real situation than the traditional 0 and 1 membership degrees. If we conduct multiple surveys and multiperson experience, the degree of approximation will be better [21].

3.2. Fuzzy Statistical Method. This method takes $n$ experts under investigation as the research object, and it forms the expert group $N$. For a known element $u$, the membership function $\mu_{A_{i}}(\mu)(i=1,2, \ldots, m)$ can be obtained by determining the multiphase fuzzy statistics of the element for $A_{1}, A_{2}, \ldots, A_{m}$ of $m$ fuzzy sets. For a single expert $P \in N$, it can be determined as follows:

$$
\begin{aligned}
\mu_{A_{i}}^{P} & = \begin{cases}1 & u \in A_{i}, \\
0 & u \notin A_{i},\end{cases} \\
\sum_{i=1}^{m} \mu_{A_{i}}^{P} & =1 .
\end{aligned}
$$

The member function $\mu$ must and only belong to one of the sets $A_{1}, A_{2}, \ldots, A_{m}$. The degree of members $\mu$ belonging to the fuzzy set $A_{i}$ can be expressed using the following equation:

$$
\mu_{A_{i}}(\mu)=\frac{\sum_{p=1}^{n} \mu_{A_{i}}^{P}}{n}
$$

The weighted average can be computed as follows:

$$
\mu_{A_{i}}(\mu)=\frac{\sum_{p=1}^{n} \bar{w}_{i} \mu_{A_{i}}^{P}}{n}
$$

3.3. Transformation Method. Conventional comprehensive evaluation techniques use simple numerical representation and the total score method or weighted summation to obtain evaluation results [22]. Compared with the traditional comprehensive evaluation techniques, the fuzzy 
comprehensive evaluation establishes the factor set $U=\left(U_{1}, U_{2}, \ldots, U_{n}\right)$ of the evaluation index and then obtains the fuzzy comment matrix through expert evaluation and other methods.

$$
R=\left(r_{i j}\right)_{n \times m} .
$$

Finally, the fuzzy transformation is performed by a suitable fuzzy calculation factor to obtain the evaluation result. For instance, the mapping shown in the following equation is called the fuzzy transformation from $U$ to $V$ :

$$
\left\{\begin{array}{l}
T_{R}: F(U) \longrightarrow F(V), \\
A: B=T_{R}(A),
\end{array}\right.
$$

where $\forall A \in F(U)$ is the original image of $B$, and $B$ is also called the image of $A$ in the case of fuzzy transformation $T_{R}$. When we assume $A: B \in F(U)$, if the transformation $T_{R}: F(U) \longrightarrow F(V)$ satisfies the following conditions in the following equation, the transformation $T_{R}$ is called a fuzzy linear transformation:

$$
\left\{\begin{array}{l}
T_{R}(A \cup B)=T_{R}(A) \cup T_{R}(B), \\
T_{R}(\lambda A)=\lambda T_{R}(A), \lambda \in[0,1] .
\end{array}\right.
$$

If $R \in F(U \times V)$ is assumed to be a given fuzzy relationship, then $R$ uniquely determines the fuzzy linear transformation relationship from $U$ to $V$. For $T_{R}: F(U) \longrightarrow F(V)$, if $\forall A \in F(U)$, then there is

$$
T_{R}(A)=A \circ R \in F(V) .
$$

When the index factor set $U=\left(U_{1}, U_{2}, \ldots, U_{n}\right)$ and the judgment set $v=\left(v_{1}, v_{2}, \ldots, v_{m}\right)$ are both finite universes, $A$ is the fuzzy vector in $U ; a=\left(a_{1}, a_{2}, \ldots, a_{m}\right)$ and $R=\left(r_{i j}\right)_{n \times m}$ are the fuzzy relationship matrix on $U \times V$, and $a \geq 0, r_{i j} \leq 1$. The fuzzy vector $B$ calculated according to the fuzzy transformation is as follows:

$$
B=A \circ R=\left(a_{1}, a_{2}, \ldots, a_{m}\right) \circ\left[\begin{array}{cccc}
r_{11} & r_{12} & \cdots & r_{1 m} \\
r_{21} & r_{22} & \cdots & r_{2 m} \\
\vdots & \vdots & \vdots & \vdots \\
r_{n 1} & r_{n 2} & \cdots & r_{n m}
\end{array}\right] \text {. }
$$

When using the fuzzy analytic hierarchy process to evaluate the state, generally, the bottom index evaluation is first performed, and then the comprehensive performance evaluation is performed through the fuzzy transformation theory. The specific steps are as follows:

(i) The establishment of the bottom-level evaluation index factor set $U=\left(U_{1}, U_{2}, \ldots, U_{m}\right)$

According to the evaluation index system model, the lowest evaluation index set is $U=\left(U_{1}, U_{2}, \ldots, U_{m}\right)$. The index of the factor set $U$ has different importance relative to the evaluation index, and the weight vector of each index needs to be formed into a fuzzy vector, which is expressed as $A=\left(a_{1}, a_{2}, \ldots, a_{m}\right)$ and used to represent the degree of importance between the indexes. (ii) The establishment of the evaluation set $v=\left(v_{1}, v_{2}, \ldots, v_{m}\right)$

In the evaluation, a unified fuzzy evaluation set must be established for both individual and overall evaluation indicators, so that the evaluation results can be accurately represented on the evaluation set.

(iii) The establishment of the bottom-level indicator comment matrix

According to the evaluation set $v=\left(v_{1}, v_{2}, \ldots, v_{m}\right)$, a mapping relationship $R: U \longrightarrow F(V)$ from $U$ to $F(V)$ is established for the evaluation of a single indicator:

$$
u_{i}: R\left(u_{i}\right)=\frac{r_{i 1}}{v_{1}}+\frac{r_{i 2}}{v_{2}}+\cdots+\frac{r_{i m}}{v_{m}} .
$$

The above formula is the fuzzy comment of the $i^{\text {th }}$ index. When the fuzzy evaluation of all indicators is performed, the fuzzy evaluation matrix $U \times V$ can be obtained, namely,

$$
R=\left[\begin{array}{cccc}
r_{11} & r_{12} & \cdots & r_{1 m} \\
r_{21} & r_{22} & \cdots & r_{2 m} \\
\vdots & \vdots & \vdots & \vdots \\
r_{n 1} & r_{n 2} & \cdots & r_{n m}
\end{array}\right] .
$$

From the above analysis, it can be seen that $(U, V, R)$ constitutes a single-layer fuzzy comprehensive evaluation model.

(iv) Comprehensive assessment of the status

According to the fuzzy vector $A=\left(a_{1}, a_{2}, \ldots, a_{m}\right)$ on the factor set $U$ and the fuzzy comment set matrix $R$, the fuzzy comprehensive evaluation vector $B=\left(b_{1}, b_{2}, \ldots, b_{m}\right)$ is obtained through proper selection of the fuzzy calculation operator and fuzzy transformation. According to the principle of maximum membership degree, the state evaluation result can be determined [23]. The comprehensive evaluation equation is as follows:

$$
\begin{aligned}
B & =A \circ R=\left(a_{1}, a_{2}, \ldots, a_{m}\right) \circ\left[\begin{array}{cccc}
r_{11} & r_{12} & \cdots & r_{1 m} \\
r_{21} & r_{22} & \cdots & r_{2 m} \\
\vdots & \vdots & \vdots & \vdots \\
r_{n 1} & r_{n 2} & \cdots & r_{n m}
\end{array}\right] \\
& =\left[b_{1}, b_{2}, \ldots, b_{m}\right],
\end{aligned}
$$

where $\circ$ is a fuzzy calculation operator, which usually contains the following calculation forms:

(a) The main factor prominent type $(M(\wedge, \vee))$. This model is characterized by ignoring other relative indicators and highlighting the main evaluation factors in the indicators. This is computed as follows 


$$
b_{j}=\bigvee_{i=1}^{n}\left(a_{i} \wedge r_{i j}\right)
$$

(b) Comprehensive control type $(M(\wedge, \otimes))$. This model revises the original index $r_{i j} r_{i j}^{a_{i}}$ to have a restrictive effect. Contrary to type $M(\wedge, \vee)$, this model highlights the secondary factors in the index. It is calculated as follows:

$$
b_{j}=\bigwedge_{i=1}^{n}\left(r_{i j}^{a_{i}}\right) \text {. }
$$

(c) Weighted average type $(M(\otimes, *))$. The contribution rate of each indicator in the model to the evaluation target is characterized by the weight and is computed as follows:

$$
b_{j}=\sum_{i=1}^{n} a_{i} r_{i j} .
$$

In the analytic hierarchy process, it is divided into several levels of evaluation index system, and multilevel fuzzy comprehensive evaluation is required [24]. When it is assumed that the $n$ subfactors of the $i^{\text {th }}$ layer constitute the corresponding factor set, the fuzzy synthesis operation combined with the fuzzy comment matrix $R_{i}$ is computed as follows:

$$
\begin{aligned}
B_{i}= & w_{i} \circ R_{i}=\left(w_{i 1}, w_{i 2}, \ldots, w_{i n}\right) \\
& \circ\left[\begin{array}{cccc}
r_{11}^{(i)} & r_{12}^{(i)} & \cdots & r_{1 m}^{(i)} \\
r_{21}^{(i)} & r_{22}^{(i)} & \cdots & r_{2 m}^{(i)} \\
\vdots & \vdots & \vdots & \vdots \\
r_{n 1}^{(i)} & r_{n 2}^{(i)} & \cdots & r_{n m}^{(i)}
\end{array}\right] \\
& =\left[b_{i 1}, b_{i 2}, \ldots, b_{i m}\right]
\end{aligned}
$$

where $W_{i}$ is the weight vector set, and $B_{i}$ is the fuzzy comment vector of the $(i+1)^{\text {th }}$ layer evaluation index of the fuzzy factor set obtained by multilayer operation.

\section{Information Dissemination Control Algorithm}

For large-scale wireless sensor networks, sensor nodes are generally randomly deployed in a target area, which can lead to irregular deployment of nodes and increase the network cost. Thus, node deployment approaches play a vigorous role under these conditions. Coverage control mostly emphasizes improving nodes deployment algorithms and correcting nodes positions to construct a network with limited resources; it also guarantees the quality of service (QoS) in monitoring region of interest (RoI) [23]. However, in conventional node deployment algorithms, the sensor nodes are arbitrarily deployed in the RoI, and this can cause the whole coverage problem, which will affect network performance. Thus, effective node deployment algorithms are essential to solve this problem.

Since there may be holes in the network connectivity of wireless sensor networks, which are caused by the exhaustion of energy of some nodes or virus attacks during the initial deployment of sensors, some areas of the network may not be reachable through the hop-by-hop propagation between sensor nodes. Therefore, static patch distribution may not be able to repair all the infected sensor nodes. However, the mobile-assisted patch distribution mechanism can overcome the problem that the network may have independent infected network areas. Therefore, the mobileassisted patch distribution mechanism can ensure that all infected sensor nodes in the network can be repaired and provide better performance in terms of repair thoroughness. Figures 1 and 2 show the difference between static patch distribution and mobile-assisted patch distribution, respectively.

When it is required to solve the number of sensor nodes in the convex hull, only the convex hull area $S$ can be solved. As shown in Figure 3, the convex hull is divided into $N-2$ triangles. When the coordinates of the three vertices of the triangle are known, the area of the triangle can be computed.

The social network can be taken as a directed graph $G=(V, E)$, where $V$ denotes the nodes in the graph, which represent the users in the social network, and $E$ denotes the edges, which represent the relationship between the users. In this context, the relationship would be that of the influencer and influenced node. Figure 4 is a state transition diagram of the information dissemination model of social networks. Each box represents the state of the node, and the arrow indicates the probability of state transition after a period of propagation, and the probability of turning into an $\mathrm{R}$ state node after losing interest, and $\mathrm{R}$ represents an E state node.

Based on the communication model constructed above, a small sample is demonstrated here, so that the process of information dissemination and transformation can be understood more clearly. Figure 5 shows a small network consisting of digging a node and 15 edges.

The above algorithm is simply applied to social networks and the Internet. This simple social network is a part of the real social network, as shown in Figure 6. The execution process and results in this kind of network are analyzed separately.

Figure 7 shows an example of a network with a community structure, where node 2 (hexagon) in community $\mathrm{C} 1$ has many connections because it is a central node, and it is marked as an important node. When the information is disseminated in the $\mathrm{C} 1$ community, node 2 will play the main role. If node 2 is immunized, the dissemination of information in the $\mathrm{C} 1$ community will be greatly affected and will be slowed down. Likewise, node 12 (square) in community $\mathrm{C} 2$ has more edges (bridge nodes) connected to the nodes in other communities. When information is disseminated among communities, node 12 plays an important role. If it is immunized, it will slow down the spread of information from community $\mathrm{C} 2$ to other communities. Therefore, both the bridge node and the community center node play an important role in the information dissemination process, and immunizing them will slow down the dissemination of all information in the network. 


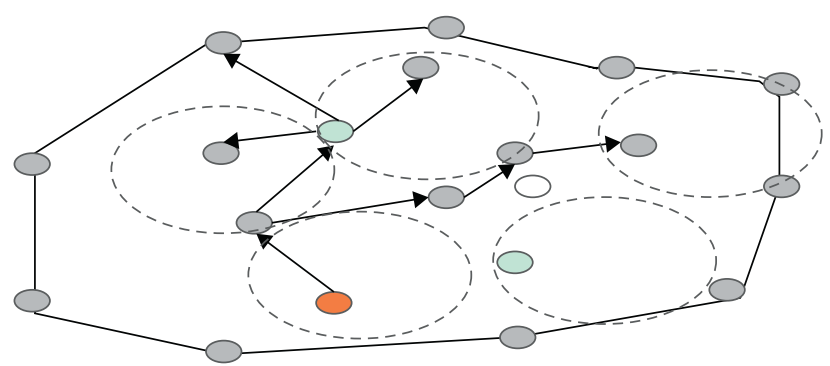

Infected nodes

Health node

Mobile indefinite distributor

FIGURE 1: Static patch distribution.



Figure 2: Mobile-assisted patch distribution.



Figure 3: Schematic diagram of convex hull segmentation.

Based on the above discussion, in this study, an improved information traceability model is proposed, as shown in Figure 8. The model contains five steps: building a network, disseminating information, deploying observation points, detecting information sources, and excluding

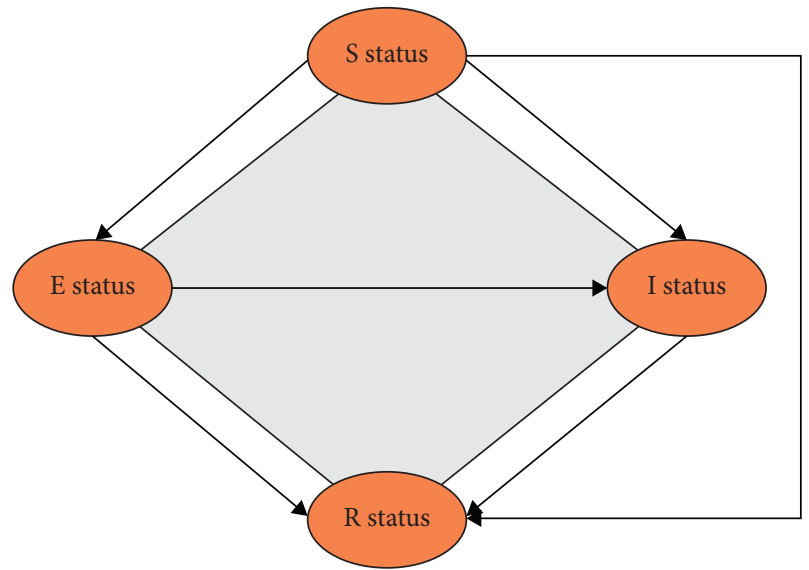

Figure 4: Each state transition.



Figure 5: A small social network.

observation points. The model can be effectively used to trace information being disseminated in a network.

Based on the fuzzy control algorithm for information traceability and dissemination, a new model is constructed for the influencing factors of information interaction between enterprises and users under the new media environment. Figure 9 shows the new information dissemination model for factors affecting information interaction. The model includes five potential variables of external factors, namely, the quality of new media platforms, the quality of new media information, the quality of new media services, the immersive experience of new media, and the user's cognition. Moreover, it also includes two internal latent variables, namely, user satisfaction with new media information interaction and using new media information interaction behavior. 


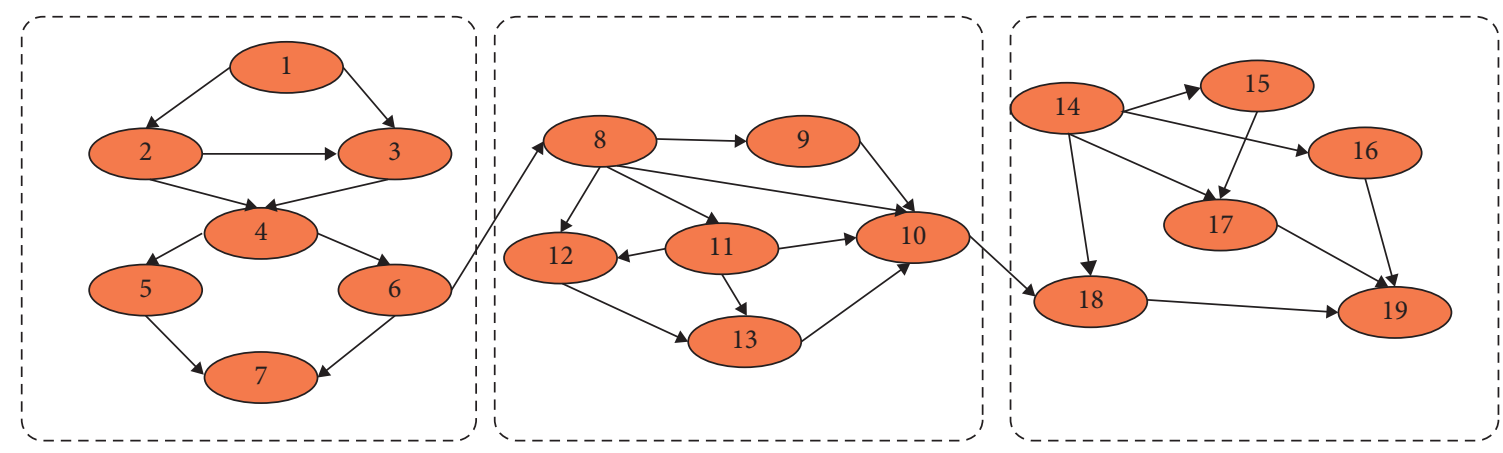

FIgURE 6: Structure diagram of the single social network.

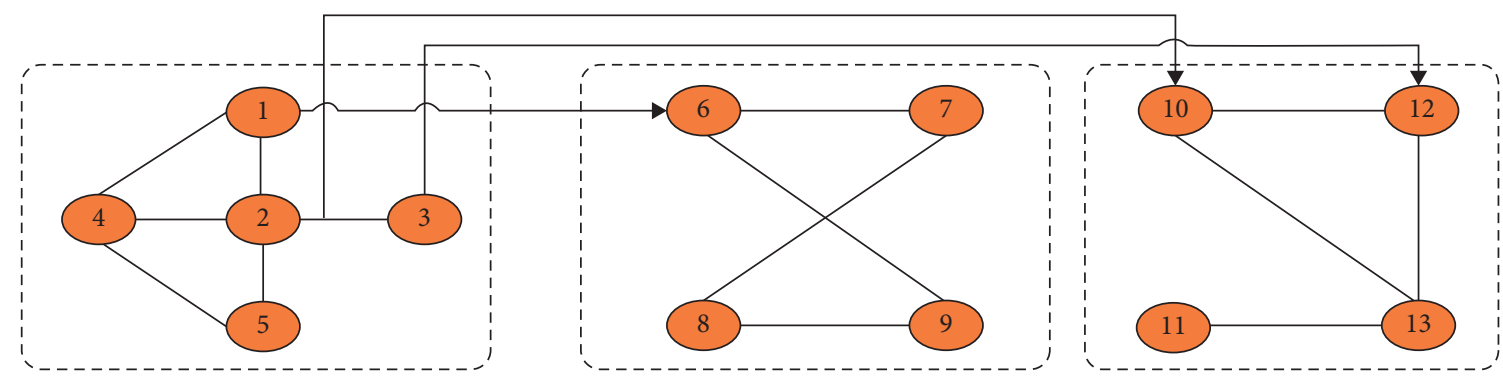

Figure 7: Central node and bridge node.

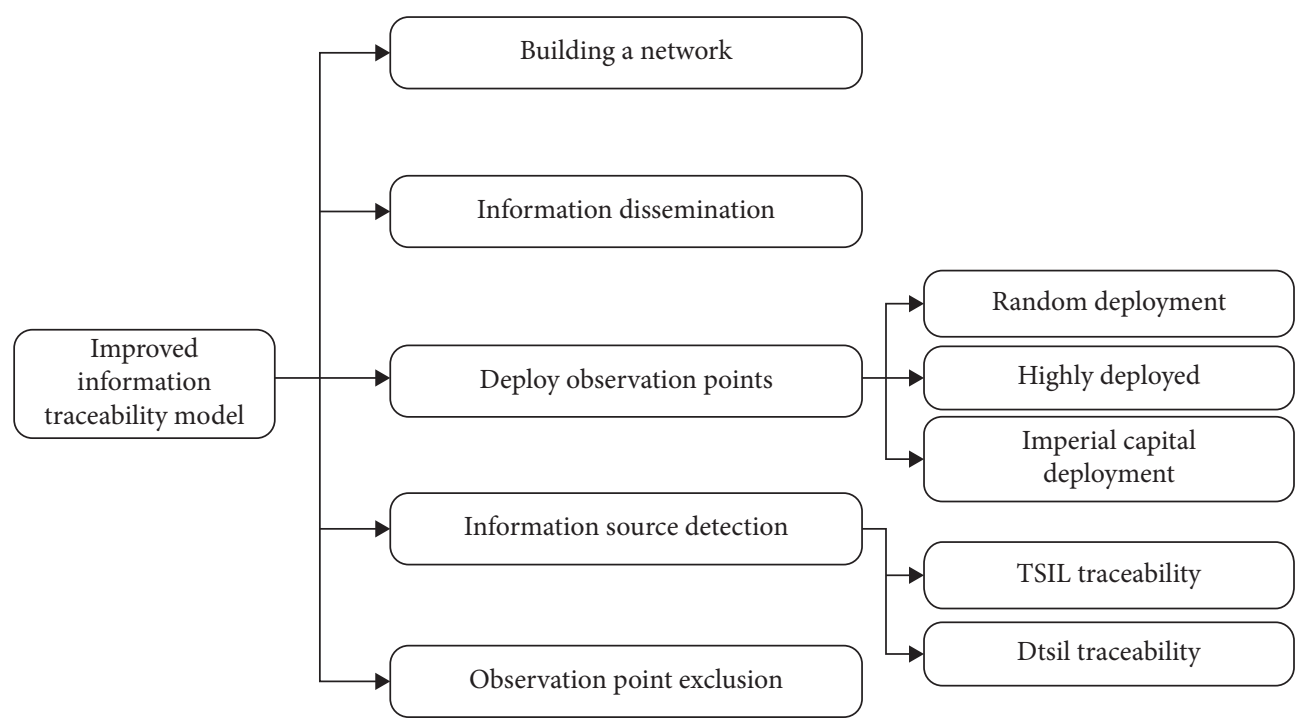

FiguRE 8: Information traceability model.

\section{Performance Evaluation}

Social networks have become the most important means of communication at present for sharing awareness, information, and innovations. With the realization that even weak social ties have the power to influence, social networks have become a medium for marketing and the influence maximization technique. This study provides an improved information dissemination control algorithm of ecological changes in the new media environment and verifies the performance of the algorithm through simulation. The proposed algorithm can effectively control the information dissemination and obtain a large number of nodes from the network. Although the locality of selected nodes cannot fully represent all social networks, the selected nodes are randomly distributed. Therefore, the selected samples of social networking sites are general and can be used to analyze social networks. The control effect and the node control effect of the algorithm were simulated. The statistical results of the simulation are represented in 


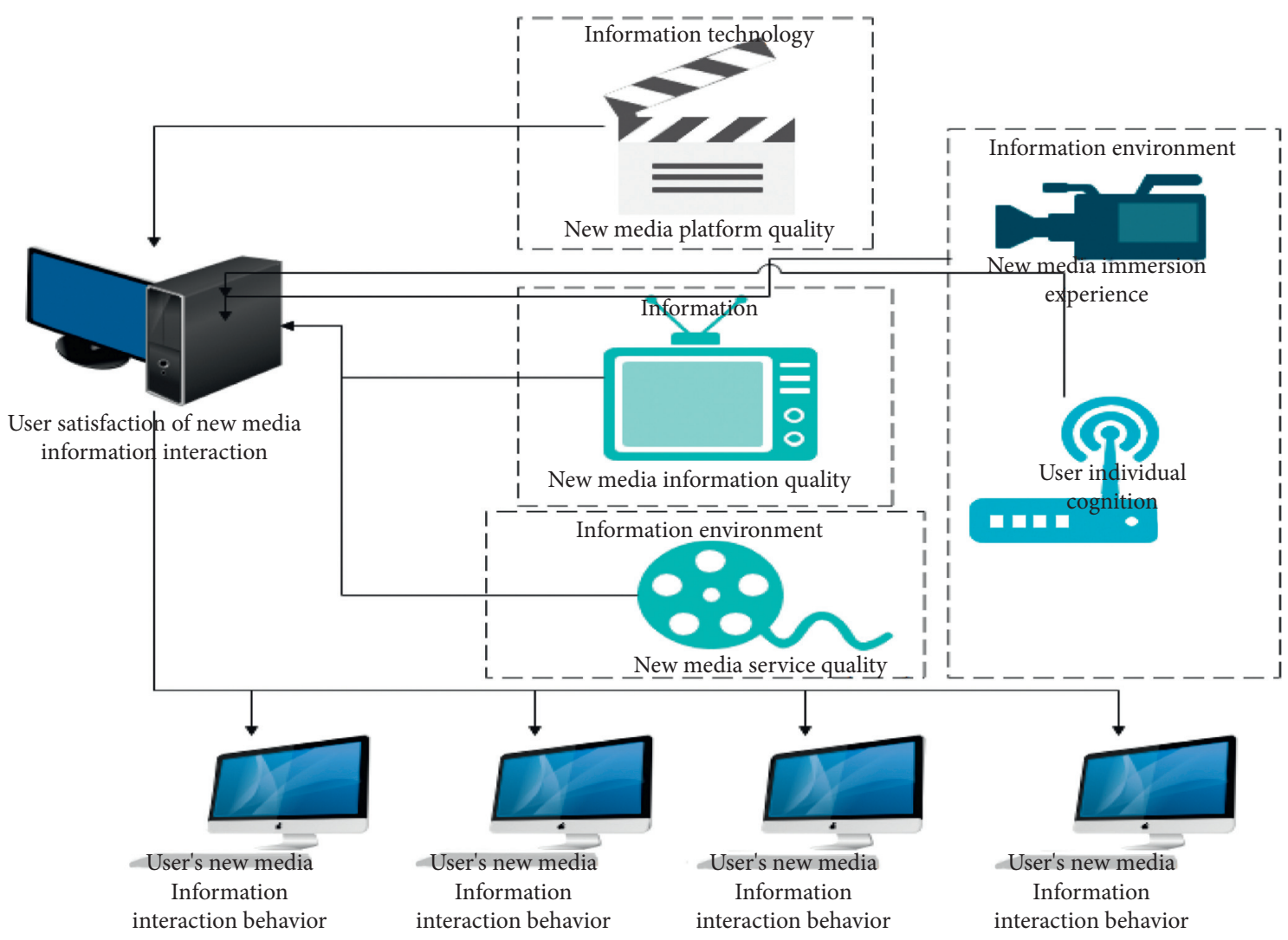

FIGURE 9: Theoretical model of factors affecting information interaction willingness.

TABLE 1: Statistical effectiveness of the information dissemination control algorithm.

\begin{tabular}{|c|c|c|c|c|c|c|c|}
\hline Number & Control score & Number & Control score & Number & Control score & Number & Control score \\
\hline 1 & 0.611 & 16 & 2.227 & 31 & 4.038 & 46 & 4.402 \\
\hline 2 & 0.818 & 17 & 2.436 & 32 & 4.074 & 47 & 4.422 \\
\hline 3 & 0.827 & 18 & 2.671 & 33 & 4.091 & 48 & 4.516 \\
\hline 4 & 0.835 & 19 & 2.802 & 34 & 4.094 & 49 & 4.519 \\
\hline 5 & 1.333 & 20 & 3.138 & 35 & 4.100 & 50 & 4.521 \\
\hline 6 & 1.335 & 21 & 3.139 & 36 & 4.100 & 51 & 4.536 \\
\hline 7 & 1.418 & 22 & 3.241 & 37 & 4.111 & 52 & 4.690 \\
\hline 8 & 1.480 & 23 & 3.420 & 38 & 4.130 & 53 & 4.736 \\
\hline 9 & 1.525 & 24 & 3.508 & 39 & 4.132 & 54 & 4.765 \\
\hline 10 & 1.538 & 25 & 3.689 & 40 & 4.198 & 55 & 4.787 \\
\hline 11 & 1.729 & 26 & 3.762 & 41 & 4.198 & 56 & 4.868 \\
\hline 12 & 1.734 & 27 & 3.830 & 42 & 4.229 & 57 & 4.869 \\
\hline 13 & 2.081 & 28 & 3.973 & 43 & 4.291 & 58 & 4.891 \\
\hline 14 & 2.092 & 29 & 4.002 & 44 & 4.316 & 59 & 4.911 \\
\hline 15 & 2.149 & 30 & 4.022 & 45 & 4.390 & 60 & 4.969 \\
\hline
\end{tabular}

Table 1 and Figure 10. The proposed algorithm can effectively improve the ecological effect of the system for the information dissemination control of ecological changes in the new media environment. The results show that as the number of nodes increases, the control score and performance of the algorithm increases. A linear trend was observed between the number of nodes and the control score. For example, for 5 nodes, the algorithm scored 1.3, and when the number of nodes is increased to 60 , the algorithm scored 4.9. Therefore, the proposed intelligent algorithm can meet the ecological information control needs of new media dissemination. 


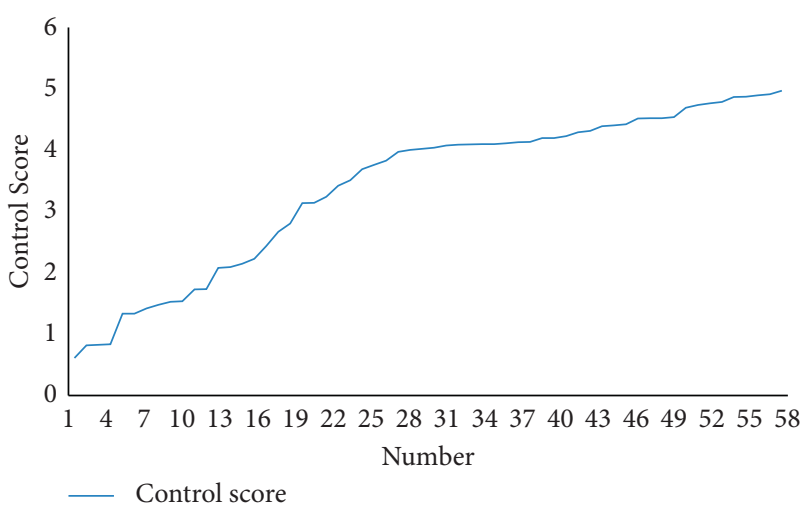

Figure 10: Statistical diagram of the effectiveness of the information dissemination control algorithm.

\section{Conclusion}

The present paper studied the information dissemination control algorithm of the ecological changes in the new media communication environment and analyzed it according to the community network model and the network communication situation in the new media era. In order to improve the effectiveness of information dissemination control of ecological change in the current media communication environment, this paper combined the current network information dissemination methods and analyzed the community network model and the network dissemination states for spreading information. We proposed an improved algorithm for information dissemination in the new media dissemination environment. The fuzzy control algorithm was employed as the core algorithm to improve and optimize the information dissemination network model. The mobileassisted patch distribution mechanism was used to repair all the infected nodes in the communication network. The simulation results showed that the proposed algorithm can effectively control the information dissemination and obtain a large number of nodes from the network. The proposed study has the potential to control effectively the information dissemination in the new media and social networks.

\section{Data Availability}

The data used to support the findings of this study are available from the corresponding author upon request.

\section{Conflicts of Interest}

The author declares that there is no conflict of interest.

\section{References}

[1] B. Y. Korniyenko and L. P. Galata, "Design and research of mathematical model for the information security system in a computer network," Наукоємні Технологіï, nо. 2, pp. 114-118, 2017.

[2] K. Hwang and M. Choi, "Effects of innovation-supportive culture and organizational citizenship behavior on e-government information system security stemming from mimetic isomorphism," Government Information Quarterly, vol. 34, no. 2, pp. 183-198, 2017.

[3] S. Chatterjee, A. K. Kar, and M. P. Gupta, "Alignment of IT authority and citizens of proposed smart cities in India: system security and privacy perspective," Global Journal of Flexible Systems Management, vol. 19, no. 1, pp. 95-107, 2018.

[4] A. Blagorazumov, P. Chernikov, and G. Glukhov, "The background to the development of the information system for aviation security oversight in Russia," International Journal of Mechanical Engineering \& Technology, vol. 9, no. 11, pp. 341-350, 2018.

[5] M. K. Özlen and I. Djedovic, "Online banking acceptance: the influence of perceived system security on perceived system quality," Accounting and Management Information Systems, vol. 16, no. 1, pp. 164-178, 2017.

[6] S. Dotsenko, O. Illiashenko, S. Kamenskyi et al., "Integrated Security management system for enterprises in industry 4.0," Information \& Security, vol. 43, no. 3, pp. 294-304, 2019.

[7] J. S. More and C. Lingam, "A SI model for social media influencer maximization," Applied Computing and Informatics, vol. 15, no. 2, pp. 102-108, 2019.

[8] D. Kemp, J. Kleinberg, and E. Tardos, Maximizing the Spread of Influence through a Social Network, SIGKDD'03, Washington, DC, USA, 2003.

[9] M. Kimura and K. Saito, "Tractable models for information diffusion in social networks," in Proceedings of the 10th European Conference on Principles and Practice of Knowledge Discovery in Databases, pp. 259-271, Berlin, Germany, September 2006.

[10] C. Zhou and L. Guoa, "A note on influence maximization in social networks from local to global and beyond," Procedia Computer Science, vol. 30, pp. 81-87.

[11] V. Yalavarthi and A. Khan, "Fast influence maximization in dynamic graphs: a local updating approach," 2018, https:// arxiv.org/pdf/1802.00574.pdf.

[12] P. Pazuraa, J. Jankowskia, and K. Bortkoa, "Modeling performance of limiting information spreading processor under competing linear threshold model," Procedia Computer Science, vol. 176, pp. 3751-3760, 2020.

[13] A. Goyal, W. Liu, and L. Laskmanan, "CELF++: optimizing the greedy algorithm for influence maximization in social networks," ACM, 2011.

[14] M. Kimura, K. Saito, R. Nakano, and H. Motoda, "Extracting influential nodes on a social network for information diffusion," Data Min Knowl Disc, pp. 70-97, Springer, Berlin, Germany, 2009.

[15] J. Leskovec, J. Kleinberg, and C. Faloutsos, "Graph evolution: densification and shrinking diameters," ACM Transactions on Knowledge Discovery from Data, vol. 1, no. 1, 2007.

[16] D. Chen, L. Lu, M. Shang, Y. Zhang, and T. Zhou, "Identifying influential nodes in complex networks," Applied Mechanics, pp. 47-55, 2011.

[17] L. Tang and H. Liu, Leveraging Social Media Networks for Classification, Data Mining, and Knowledge Discovery, Springer, Berlin, Germany, 2011.

[18] L. Liu, J. Tang, J. Han, and S. Yang, "Learning influence from heterogeneous social networks," Data Mining and Knowledge Discovery, pp. 511-544, Springer, Berlin, Germany, 2012.

[19] Y. Y. Yu, F. Jiang, J. Du, and D. Gong, "SVMs classification based two-side cross-domain collaborative filtering by inferring intrinsic user and item features," Knowledge-Based Systems, vol. 141, pp. 80-91, 2018.

[20] B. Wang, X. Gu, and L. Ma, "Temperature error correction based on BP neural network in meteorological wireless sensor 
network," International Journal of Sensor Networks, vol. 23, no. 4, pp. 265-278, 2017.

[21] J. Zhang, J. Tang, and T. Wang, "Energy-efficient datagathering rendezvous algorithms with mobile sinks for wireless sensor networks," International Journal of Sensor Networks, vol. 23, no. 4, pp. 248-257, 2017.

[22] Y. Yu, F. Jiang, J. Du, and D. Gong, "A cross-domain collaborative filtering algorithm with expanding user and item features via the latent factor space of auxiliary domains," Pattern Recognition, vol. 94, pp. 96-109, 2019.

[23] H.-J. Zimmermann, "Fuzzy set theory," Wires Computational Statistics, vol. 2, no. 3, pp. 317-332, 2010.

[24] M. Yu, T. Quan, Q. Peng, X. Yu, and L. Liu, "A model-based collaborate filtering algorithm based on stacked AutoEncoder," Neural Computing \& Applications, 2021. 